\title{
Quantum Locking and the Meissner Effect Lead to the Origin and Stability of the Saturn Rings System
}

\author{
Vladimir V. Tchernyi (Cherny), Andrew Yu. Pospelov \\ Modern Science Institute, SAIBR, Moscow, Russia \\ Email: chernyv@bk.ru
}

How to cite this paper: Tchernyi (Cherny), V.V. and Pospelov, A.Yu. (2018) Quantum Locking and the Meissner Effect Lead to the Origin and Stability of the Saturn Rings System. International Journal of Astronomy and Astrophysics, 8, 104-120. https://doi.org/10.4236/ijaa.2018.81008

Received: January 30, 2018

Accepted: March 26, 2018

Published: March 29, 2018

Copyright ( 92018 by authors and Scientific Research Publishing Inc. This work is licensed under the Creative Commons Attribution International License (CC BY 4.0).

http://creativecommons.org/licenses/by/4.0/

\begin{abstract}
It is demonstrated how superconducting iced particles of the protoplanetary cloud of Saturn are coming to magnetic equator plane and create the stable enough rings disk. There are two steps. First, after appearance of the Saturn magnetic field due to Meissner phenomenon, all particles orbits are moving to the magnetic equator plane. Finally they become distributed as rings and gaps like iron particles around magnet on laboratory table. And they are separated from each other by the magnetic field expelled from them. It takes up to few tens of thousands years with ten meters rings disk thickness. Second, because of quantum locking all particles become to be locked within magnetic well at the magnetic equator plane due to Abrikosov vortex for superconductor. Finally each particle is locked within three-dimensional magnetic well. It works even when particles have small fraction of superconductor. During the rings evolution some contribution to the disk also could come from the collision-generated debris of the current moon, coming meteorites and from the geysers like it happened due to magnetic coupling of Saturn and Enceladus. The rings are relict of the early days of the magnetic field of Saturn system.
\end{abstract}

\section{Keywords}

Origin of Saturn Rings, Age of Saturn Rings, Superconductivity of Saturn

Rings, Quantum Locking of Saturn Rings, Space Superconductivity,

Quantum Phenomena in Space

\section{Introduction}

Since 1610 when Galileo Galilei discovered such a nice and attractable space planetary structure there has not been yet complete understanding of the Saturn rings origin and age. There are three version of this process. Two of them have 
very close ideas of the gravity defragmentation when massive object was tidally disrupted when passing nearby Saturn [1] [2]. It was postulated that the rings particles can be collision-generated debris of this massive object. One well-developed theory is saying that an asteroid was destroyed coming to the Roche zone [1]. This approach was criticized because it does not have conformation of Cassini probe due to the fact that iced particles of the rings do not have iron which is contained in the asteroid matter. Note that metal matter of asteroid as a heavy part may disappear by going to the Saturn by gravity. Later theory suggested a Titan-sized satellite was destroyed by passing Saturn as it migrates inward towards Saturn [2]. Here it is not clear well where the core of it disappeared.

Also we could not consider both theories as eventually completed. Because, for example, they could not explain particles separation, thin width and sharp edges of the rings, why rings exist only for the planets behind the asteroid belt, electromagnetic phenomena of the rings, spokes in the $B$ ring, and other phenomena.

There is no yet clear answer about the age of the rings formation. It remains a mystery. First idea was that they were as old as the Saturn system. Then after measurements of meteoroid flux into the Saturn system from Cassini's dust-counting instrument since the spacecraft arrived at Saturn in 2004, Kempf and colleagues showed that the still-bright rings collect too much dust pollution to have maintained their youthful shine for billions of years [3]. Authors concluded the ice of the rings should be far dirtier than it was if it had been gathering space dust during 4 billion years and rings disk may be much younger than Saturn system itself.

But when the Cassini spacecraft sent back the clearest images yet of Saturn's rings, M. Hedman and P. Nicholson [4] have received a totally new understanding of what is the matter of the $B$ ring system of Saturn. After studying photos images, they came to the conclusion that the most striking $B$ ring of Saturn's demonstrates for the observer an optical illusion. The mass of the $B$ ring is only from one-half to one-seventh of the previously existing assessment. Those measurements reveal that the $B$ ring, which makes up 80 percent of the total ring mass, is about 0.4 times that of Saturn's moon Mimas. In fact, $B$ ring of Saturn contain three times less matter than previously thought. "The best analogy of this situation with $B$ ring of Saturn is something like a fog over the meadow may seem less transparent and empty, than a water-filled pool, which has a much higher density than the fog" (P. Nicholson). And authors conclude that rings are much younger.

For example the guess of L. Esposito is that a single moon about half the mass of Mimas was ripped up around 200 million years ago [5]. It means Saturn's rings are surprisingly young and may be from shredded moons. Also more detailed discussion about age and fate of Saturn rings was presented J. Henry [6]. It looks like the best answer of the problem with the ring origin and age is given by the title of the paper written by P. Estrada, R.H. Durisen and J.N. Cuzzi: “After 
the Cassini grand finale, is there a final consensus on ring origin and age?" [7]. Also it was discussed frequently, for example in [8] [9] [10] [11] [12].

We have noted that all discussed above models of the origin and age of Saturn rings are based on classical understanding of the gravitational defragmentation of the massive body [13]. But both above theories do not allow a single common position of the physical concept in order to extend our understanding of many experimentally observed effects of the rings. And there has not been yet a clear explanation why and how particles rotating around Saturn on chaotic orbits within protoplanetary cloud coming to the equator rings disk and are held inside of it for a long time.

Our third version of the rings origin is also related to the Safronov theory of planet formation and it is based on the evolution of the protoplanetary cloud [13]. At the beginning of the process of formation of the rings system all iced particles are rotating around Saturn on chaotic orbits inside the protoplanetary cloud in accordance with the Kepler's law. After appearance of the magnetic field of Saturn all iced particles start to interact with it. The question is how all orbits of the particles may come to the equatorial plane of Saturn. If we are able to find answer to this question so it means that particles of the rings have approximately the same age as the age of the magnetic field of the Saturn, and the rings disk particles are relict of the early days of the Saturn system magnetic field.

In order to solve problem of the Saturn rings origin we suggested that iced particles of the rings may possess electromagnetic quality of superconductor [4]-[23]. The reason for this suggestion is existence of magnetic field of Saturn and low temperature in it vicinity. Electromagnetic simulation has demonstrated that during some time after appearance of the Saturn magnetic field, all the chaotic orbits of the superconducting particles of the protoplanetary cloud will go to the magnetic equator plane where the magnetic energy has a minimum value. For Saturn magnetic equator is almost coincident with the geographical equator. Finally particles become distributed as rings and gaps like iron particles around magnet on laboratory table. And particles are separated from each other by the magnetic field expelled from them. Surprisingly, assuming Saturn's rings contain superconducting matter many difficulties in understanding the nature of the rings can find simple explanations [24]-[36]:

- origin, evolution, and dynamics of the rings

- considerable flattening and the sharp edges of the ring system

- why particles of the rings are separated

- formation of "spokes" in the $\operatorname{ring} B$

- high radio-wave reflectivity and low brightness of the rings

- anomalous reflection of circularly polarized microwaves

- spectral anomalies of thermal radiation of the rings

- why planetary rings in the solar system appear only after asteroid belt

- why the Earth has no rings 
As we can see it already, the data of the Cassini mission once again confirms importance of electromagnetic interactions and the correctness of the conclusion of $\mathrm{H}$. Alfven that the "solar system history is recorded in the Saturn rings structure" [37]. And it becomes to be a physical reality of our electromagnetic model to extend common understanding of the Saturn rings origin. At the same time we still have some questions about stability of the rings disk, it thickness and time of creation. Below we try to provide some additional information related to solution of these problems. Now we are going to add our consideration of the Saturn rings origin going to the quantum understanding of the superconductivity of iced particles and influence of it on the stability of the rings disk.

\section{Discussion about Electromagnetism and Quantum Phenomenon of the Superconductor Related to Solution of the Proposed Problem}

An interesting idea of the rings formation is coming from the general electromagnetic theory physics when we notice the similarity of the picture of the Saturn rings to the picture when iron particles creates dense and rarefied regions in a nonuniform magnetic field near by the magnet on laboratory table (please, see Figure 1).

The question is how we can get the same good looking structure for the rings of Saturn located within equator plane from the situation when iced particles are moving around Saturn by chaotic orbit within protoplanetary cloud.

Recently Cassini mission has demonstrated that the rings consist of pure ice, 93\% with some carbon matter impurities, $7 \%$

(https://www.nasa.gov/mission_pages/cassini/media/cassini-090204.html). The temperature in the area of the rings is about $70-110 \mathrm{~K}$. That's why superconducting substance may be possible in space behind the belt of asteroids.

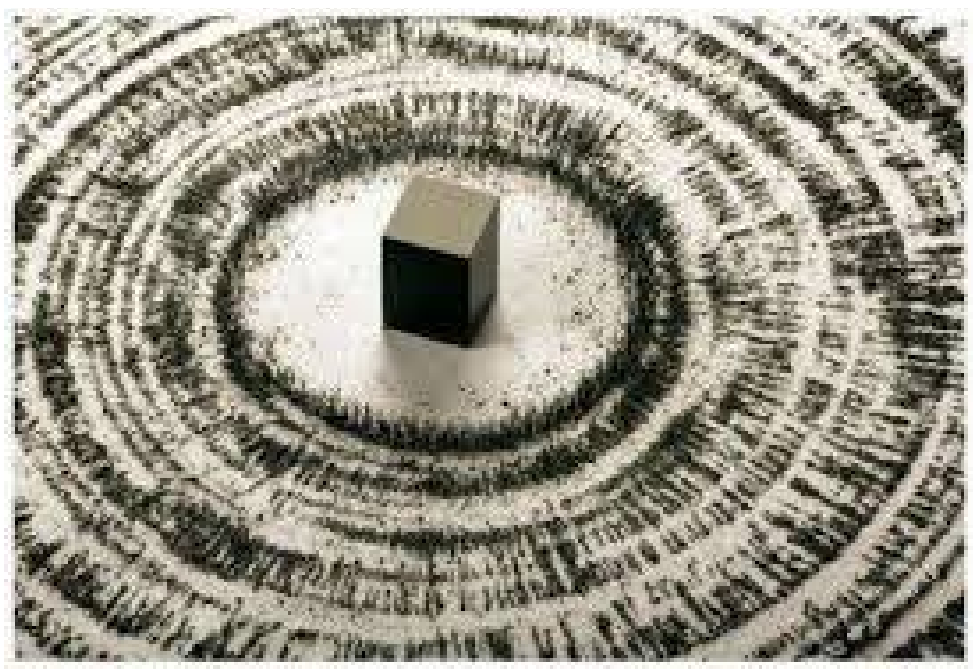

Figure 1. https://wadevenden.files.wordpress.com/2014/07/magnet.jpg?w=500 Iron particles create dense and rarefied regions in nonuniform magnetic field near by the magnet on laboratory table. 
The high-temperature superconductivity was discovered by J. G. Bednorz and K. A. Muller in 1986 [38]. Ice is a complicated matter. We do have a limited knowledge about ice in space. At the same time G.V. Babushkina et al. presented superconductivity of ice in 1986 [39]. In 2015 F. Yen and T. Gao found evidence of macroscopic quantum phenomena (superconductivity) in ice [40]. For the carbon C36 M. Cote et al demonstrated superconductivity in 1998 [41]. Recently also team of physicists has found the small amounts of superconducting materials might be relatively common in meteorites [42].

Recently was demonstrated an important fact that even small amount of superconducting matter may hold more than 70,000 times its own weight [43]. The experimental set up of how high temperature superconductivity is working on the best way B. Almog presented in Chicago at the ASTC Conference in 2011. It is based on theoretical discovery of A. Abrikosov of the vortex structure as a quantum nature of the superconductor [44]. Then U. Essmann, H. Träuble [45] and A. Tonomura [46] for the first time demonstrated experimentally and visualized it that the magnetic field may quantized inside the superconductor.

An interaction of superconductor of the second group with external magnetic field is a quantum physical phenomenon. The magnetic field inside superconductor behaves like a quantum particle, like a quantum object. And as a fact magnetic field is magnetized inside superconductor and strands of lines of the magnetic field remain inside superconductor, they gets locked inside superconductor (please, see Figure 2). Some of the flux line becomes to be pin, and they never could take move. The reason for that is that superconductor doesn't like magnetic fields lines moving around. What it actually does, it locks them in place. By doing that it blocks itself.

Phenomenon of "Abrikosov vortex" is known as the partial penetration of the magnetic field in the superconductor of the second group, which does not lead to the complete destruction of superconductivity. The volume of material is pierced by the single quanta of magnetic flux, each of which is shielded from the rest of the sample circular superconducting currents. Why it happens? Under the low temperature some of magnetic lines will be expelled outside of the sample of superconductor of the second group. Because it is not a classical Meissner phenomenon, it's a quantum phenomenon. At the same time some of lines will remain inside the sample and superconductor locks this magnetic field flux lines inside itself (please, see Figure 2(b) and Figure 2(c)).

The action of locking prevents superconducting particles from moving within the disc. It is quantum trapping, quantum locking and quantum levitation. Also the action of trapping prevents the disc itself from moving in space. It is interesting notes that almost all new superconducting compound, open from the early sixties up to the present time, are superconductors of the second group [47]. The list includes organic superconductors, fullerenes (composed of atoms of carbon) and high temperature superconductors, and others.

Flux pinning is the phenomenon where a superconductor is pinned in space above a magnet. The superconductor must be a type-II superconductor 


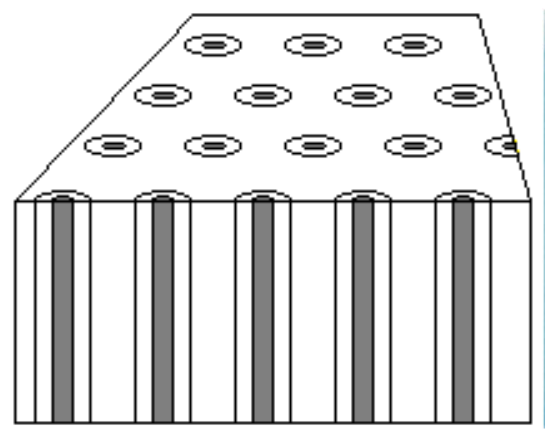

(a)

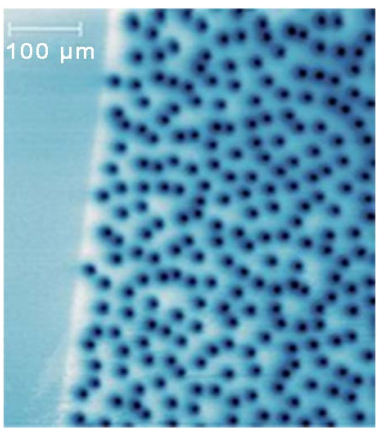

(b)

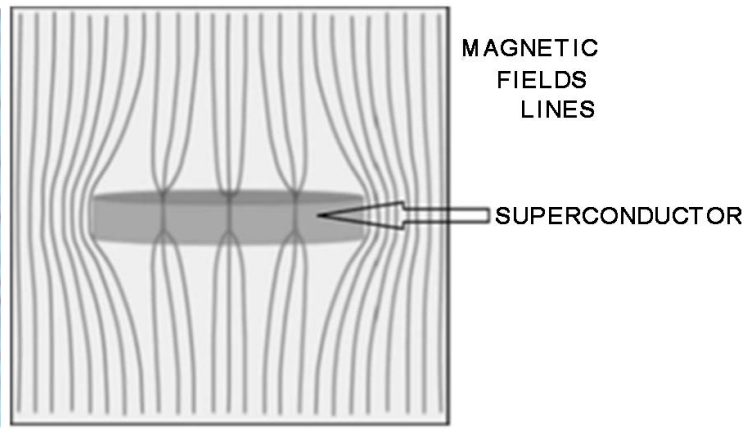

(c)

Figure 2. (a) Magnetic field is magnetized inside superconductor. Some of the flux line becomes to be pin, they don't move. This type superconductor allows only partial expulsion of a magnetic field. The volume of it is permeated with a kind of strands inside (dark lines), also known as "Abrikosov vortex" (a fluxon), where the superconductor becomes a normal conductor. But the whole sample behaves like a superconductor (http://makeitquantum.ru/superconductor-in-magnetic-field/); (b) This is a real photo of the surface of a 200-nm-thick YBCO superconducting film with flux pinning structure (vortices) taking by scanning SQUID microscopy. Huge amount of magnetic field lines penetrating sample of superconductor (vortices) and hold it against any perpendicular horizontal movements (F. S., Pan, A. V., Wang, R., Fedoseev, S. A. and Hilgenkamp, H. (2015). Analysis of low-field isotropic vortex glass containing vortex groups in $\mathrm{YBa}_{2} \mathrm{Cu}_{3} \mathrm{O}_{7-\mathrm{x}}$ thin films visualized by scanning SQUID microscopy. Scientific Reports, 5, 8677. https://www.nature.com/articles/srep08677); (c) Natural superconducting iced particle of the rings which is located along the horizontal equator plane is interacting with vertical Saturn's magnetic field lines. We can see that some of magnetic lines will be expelled outside of the sample of superconductor of the second group. And at the same time some of them will remain inside the sample and superconductor locks this magnetic field flux lines inside itself

(https://en.wikipedia.org/wiki/Flux_pinning).

(https://en.wikipedia.org/wiki/Flux_pinning). These sites of penetration are known as flux tubes. The number of flux tubes per unit area is proportional to the magnetic field with a constant of proportionality equal to the magnetic flux quantum. For example, on a simple 76 millimeter diameter, 1-micrometer thick disk, next to a magnetic field of $28 \mathrm{kA} / \mathrm{m}$, there are approximately 100 billion flux tubes that hold 70,000 times the superconductor's weight. At lower temperatures the flux tubes are pinned in place and cannot move. This pinning is what holds the superconductor in place thereby allowing it to levitate. This phenomenon is closely related to the Meissner effect, though with one crucial difference-the Meissner effect shields the superconductor from all magnetic fields causing repulsion, unlike the pinned state of the superconductor disk which pins flux, and the superconductor in place.

For the rings disk of Saturn the problem of stability of the pinning structure of the superconducting iced particles is important. An exact mathematical solution of it was presented by I.M. Sigal, T. Tzaneteas in 2013 [48] and in 2015 [49] on the bases of Ginzburg-Landau equations which play a key role in superconductivity and particle physics. These equations have two remarkable classes of solutionsvortices and (Abrikosov) vortex lattices. For the standard cylindrical geometry, the existence theory for these solutions, as well as the stability theory of vortices are well developed. The latter is done within the context of the time-dependent Ginzburg-Landau equations-the Gorkov-Eliashberg-Schmid equations of superconductivity-and the abelian Higgs model of particle physics. Authors 
study stability of Abrikosov vortex lattices under finite energy perturbations satisfying a natural parity condition (both defined precisely in the text) for the dynamics given by the Gorkov-Eliashberg-Schmid equations. For arbitrary lattice shapes, was proved that there exists a modular function depending on the lattice shape such that Abrikosov vortex lattice solutions are asymptotically stable under finite energy perturbations (defined precisely in the text), provided the superconductor is of Type II and this function is positive.

In case of Saturn rings we do have situation which looks similar to the case shown above in [43]. The rings particles contain $93 \%$ of ice and only $7 \%$ of carbon. It means that even if ice of the rings particles is not completely superconducting, still phenomenon of superconductivity will be responsible for the rings disk formation due to contains $7 \%$ of carbon. But superconductivity of ice is demonstrated in [39] and [40] and for carbon C36 in [41].

Consequently superconducting iced particles will be locked within the rings disc of the magnetic equator plane by the Saturn magnetic field due to phenomena of quantum locking, quantum trapping and quantum levitation. The rings disk itself will be suppressed by the magnetic pressure from both sides along the $\mathrm{z}$ axis, because along a meridian the magnetic energy becomes to be bigger on the distance from the minimum value at the magnetic equator. Finally we have a magnetic well of the rings disc which is sufficiently disturbing picture of Saturn magnetic field lines in the area of magnetic equator, Figure 3.

As a result each particle of the rings disk of Saturn is locked strong enough within three-dimensional magnetic well. Thus it means we have a stable enough structure of the rings disk which is stabilized by the magnetic force on the vertical direction due to the gradient of magnetic flux and on the horizontal direction by the quantum locking and quantum pinning of the Abrikosov vortex structure for each particle.

Following our superconducting model for the rings origin we are coming to new understanding of the "spokes" formation in the $B$ ring. Just as any wheel spokes, the spokes of the rings are aligned almost radially. The size of the spokes is about $10^{4} \mathrm{~km}$ along the radius and about $10^{3} \mathrm{~km}$ along the orbit of the rings. The matter of the spokes consists of micron and submicron size particles. There were many attempts to explain the nature of these spokes. Generally, all the models are based on the action of the force of gravity. A different idea was put forward that the nature of rotating spokes is related to electromagnetic force of the diamagnetic expulsion due to Meissner effect. Analysis of the spectral radiation power of spokes provides a specific periodicity about $640.6 \pm 3.5 \mathrm{~min}$ which almost coincides with the period of rotation of the magnetic field of Saturn (639.4 $\mathrm{min})$. Moreover a strong correlation of maxima and minima of activity of spokes with the spectral magnetic longitudes is connected to presence or absence of the radiation of Saturn's Kilometric Radiation (SKR). It enhances the assumption of dependence of the spokes dynamics on the magnetic field of Saturn and it testifies to the presence of large-scale anomalies in the magnetic field of Saturn. As a result we have the next explanation. 


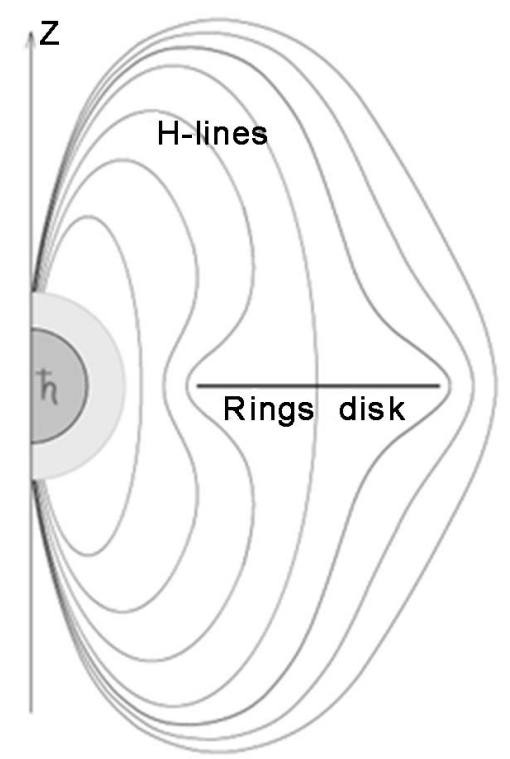

Figure 3. Thus particles within the rings disc become to be locked by the quantum locking along the horizontal line. And they also suppressed by the external magnetic field from both directions along the meridian, because the magnetic energy becomes to be bigger on the distance from the minimum value at the magnetic equator. Magnetic well of the rings disc is sufficiently disturbing picture of Saturn magnetic fields lines in the area of magnetic equator.

Superconducting ice particles which are locked off the ring matter are orbiting in accordance with the Kepler's law and they have their own speed on each orbit. Magnetic field of Saturn has its own anomalies along the radius in some directions. When the particles enter into this anomalous region, the diamagnetic expulsion force that is applied to the particles changes its value. Then particles begin to change their orbit. For the significant number of participating particles, for the external observer, this process appears as the turbulent cloud stretched along the radius in the form of spokes. During this time should happened ice particles collision. If particles approaching distance about point contact about $10^{-8} \mathrm{~m}$ then electrons can be tunneled through it. Consequently, this type of superconducting weak link begins to generate electromagnetic radiation-a non-stationary Josephson phenomenon. As a result the $B$ ring may generate wideband pulse radiation at $20 \mathrm{KHz}-40.2 \mathrm{MHz}$ related to anomaly of the Saturn magnetic field. After passing anomaly, particles return to their prior orbit and the common appearance of the rings is recovered.

\section{Movements of the Superconducting Iced Particles to the Magnetic Equator Plane}

Following our electromagnetic model prior emergence of the Saturn magnetic field all the particles within the protoplanetary cloud are located on the chaotic Keplerian orbits, exhibiting a balance of the force of gravity and the centrifugal 
force. With emergence of the Saturn magnetic field, the superconducting particles of the protoplanetary cloud begin to demonstrate an ideal diamagnetism (Meissner-Ochsenfeld phenomenon). Particles begin to interact with the magnetic field and all the particles become to be involved in an additional azimuth-orbital motion due to the Meissner effect. We try to estimate the result of this motion. We will follow procedure of electromagnetic modeling already demonstrated earlier [24]-[36]. Let's consider diamagnetic ball with mass $m$ flying in a gravitational and magnetic field of a planet with a given initial velocity and distance from the center of the planet. Find its trajectory. In the spherical coordinate system we get:

$$
r=\sqrt{x^{2}+y^{2}+z^{2}}, \varphi=\arctan (y / x), \theta=\arccos (z / r)
$$

And the acceleration vector in this coordinate system consequently is

$$
\begin{aligned}
\boldsymbol{a}= & \boldsymbol{e}_{r}\left(\ddot{r}-r \dot{\theta}^{2}-r \dot{\varphi}^{2} \sin ^{2} \theta\right)+\boldsymbol{e}_{\theta}\left(r \ddot{\theta}+2 \dot{r} \dot{\theta}-r \dot{\varphi}^{2} \sin \theta \cos \theta\right) \\
& +\boldsymbol{e}_{\varphi}(r \ddot{\varphi} \sin \theta+2 \dot{r} \dot{\varphi} \sin \theta+2 r \dot{\theta} \dot{\varphi} \cos \theta)
\end{aligned}
$$

Velocity is $\boldsymbol{v}=\dot{\boldsymbol{r}} \boldsymbol{e}_{r}+r \dot{\theta} \boldsymbol{e}_{\theta}+r \dot{\varphi} \sin \theta \boldsymbol{e}_{\varphi}$ and $\boldsymbol{r}=r \boldsymbol{e}_{r}$

The magnetic induction of a planet is

$$
\boldsymbol{B}=\frac{\mu_{0}}{4 \pi} \frac{3 \boldsymbol{r}(\boldsymbol{\mu r})-\boldsymbol{\mu} r^{2}}{r^{5}}
$$

The magnetic field is $\boldsymbol{B}=\mu_{0} \boldsymbol{H}$, and magnetization $\boldsymbol{B}=\mu_{0}(\boldsymbol{H}+\boldsymbol{M})$, $\boldsymbol{M}=\chi_{m} \boldsymbol{H}$.

We place now a diamagnetic sphere into the uniform magnetic field and see what happens.

No currents flow, then $\nabla \times \boldsymbol{H}=0$, and we introduce a scalar potential $\boldsymbol{H}=-\nabla \psi$

$\operatorname{div} \boldsymbol{B}=0$, then $\nabla^{2} \psi=0$. A uniform magnetic field is represented by its magnetic potential

$$
\begin{gathered}
\psi_{0}=-H_{0} z=-H_{0} r \cos \theta \\
\psi= \begin{cases}-H_{0} r \cos \theta+\frac{a \cos \theta}{r^{2}}, & r>R \\
-b r \cos \theta, & r<R\end{cases} \\
a=\frac{1}{3} R^{3} M, \quad b=H_{0}-\frac{1}{3} M
\end{gathered}
$$

Here $b$ is the value for the uniform magnetic field inside the particle: $M=\chi_{m} H_{\text {int }}=\chi_{m} b$.

For a superconductor, we get $\chi_{m}=-1$.

So, finally an effective magnetic dipole moment is given $\boldsymbol{m}=-2 \pi R^{3} \boldsymbol{H}_{0}$.

The potential energy of a magnetic dipole $\boldsymbol{m}$ is $U=-\boldsymbol{m} \boldsymbol{B}$. For a rigid dipole, the force then is

$\boldsymbol{F}=(\boldsymbol{m} \nabla) \boldsymbol{B}$, then we obtain $\boldsymbol{F}=-\pi \mu_{0} R^{3} \nabla H_{0}^{2}$.

In a simplified version, a magnetic moment for the particle in CGS units is $\boldsymbol{M}=-R^{3} \boldsymbol{H}$. 
We place the origin of the coordinates at the center of a planet, and the $z$ axis directly along the magnetic moment of a planet (orthogonal to equator). Then the magnetic energy will become as:

$$
U=-\mu_{p} \boldsymbol{B}=R^{3} H^{2}=\frac{R^{3} \mu^{2}}{r^{6}}\left(3 \cos ^{2} \theta+1\right)
$$

Here $\theta$-the angle between the vector $\boldsymbol{r}$ and the $z$ axis. It can be seen from this expression, that the magnetic energy of the superconducting particle has a minimum value when the radius-vector $\boldsymbol{r}$ is in a plane of the magnetic equator plane, $(\cos \theta=0)$. It means that eventually a particle during an interaction with magnetic field of planet tries to get position within magnetic equator plane and then it continues move around Saturn within the rings disk in accordance with Kepler's low [24]-[36].

Now going to spherical coordinates we get

$$
\nabla U=\frac{\partial U}{\partial r} \boldsymbol{e}_{r}+\frac{1}{r} \frac{\partial U}{\partial \theta} \boldsymbol{e}_{\theta}
$$

The Newton equation of motion is then

$$
m \boldsymbol{a}=-\left(\frac{G m M}{r^{2}}+\frac{\partial U}{\partial r}\right) \boldsymbol{e}_{r}-\frac{1}{r} \frac{\partial U}{\partial \theta} \boldsymbol{e}_{\theta}
$$

In order to do calculations we take the following values for parameters: at equator $R_{S}=60268 \mathrm{~km}, M=5.6846 \times 10^{26} \mathrm{~kg}$,

$G=6.67259 \times 10^{-11} \mathrm{~m}^{3} \cdot \mathrm{kg}^{-1} \cdot \mathrm{s}^{-2}$ and the density of an ice particle $\rho \approx 1 \Gamma / \mathrm{cm}^{3}$ The Saturn magnetic field at the equator is 0.2 gauss. Then the Saturn magnetic moment may be calculated as

$$
B=\frac{\mu_{0}}{4 \pi} \frac{\mu}{R_{S}^{3}}, \mu=\frac{4 \pi R_{S}^{3} B_{S}}{\mu_{0}}, \mu=4.378 \times 10^{25} \mathrm{~m}^{2} \cdot \mathrm{A}
$$

Calculated time to change the orbit of the particle to the arrival of it into the plane of the magnetic equator plane due to the Meissner effect depends on the parameters of the particles and the distance from the planet and it varies from several thousand years to several tens of thousands of years. During this time the additional magnetic force will modify significantly the orbit of the particle. To get thickness of the rings sombrero we consider a superconducting rings disc around a planet and evaluate a magnetic field around. Nearby Saturn the sphere has a uniform magnetization. Estimates of the thickness of the disc rings give a value of about 10 meters. This result is coincident with experimental data.

\section{Notes about Time of the Rings Disc Formation}

We made modeling for one particle. In reality time for particles to come to magnetic equator plane will be much larger due to collisions and the influence of Meissner phenomenon in the azimuth-orbital direction because magnetic field of Saturn is nonuniform. Important to remember the orbital resonances, moons and ring particles as well as mechanical, plasma and magnetohydrodynamic interactions also can play an important role in forming a specific structure of the 
rings and gaps. All these factors also may contribute to the time of the rings structure origin, dynamic and evolution of the initial origin of existing structure of the Saturn rings.

Also there is a probability of contribution to the matter of the rings disc from the debris of the asteroids, moon and satellites migrated inward towards Saturn and coming superconducting meteorites. Another situation may happen when particles of the frozen water coming from the geyser of the geologically active satellite go to the rings disc due to magnetic coupling between Saturn and its satellite (Figure 4).

An important discovery was made in 2005. The Cassini spacecraft started multiple close flybys of Enceladus, revealing its surface and environment in greater detail. In particular, Cassini discovered water-rich plumes venting from the south polar region [50]. Cryovolcanoes near the south pole shoot geyser-like jets of water vapor up to $500 \mathrm{~km}$, molecular hydrogen, other volatiles, and solid material, including sodium chloride crystals and ice particles, into space, totaling about $200 \mathrm{~kg}$ per second [51]. Over 100 geysers have been identified [52]. Some of the water vapor falls back as "snow"; the rest escapes, and supplies most of the material making up Saturn's E ring [53] [54] (Figure 4 and Figure 5). The intensity of the geysers of Enceladus depends on how close or far the satellite is from Saturn [55].

Certainly process of supplying particles to the rings disc from the geyser of the satellite of Saturn could not continue forever. It works only during the time of

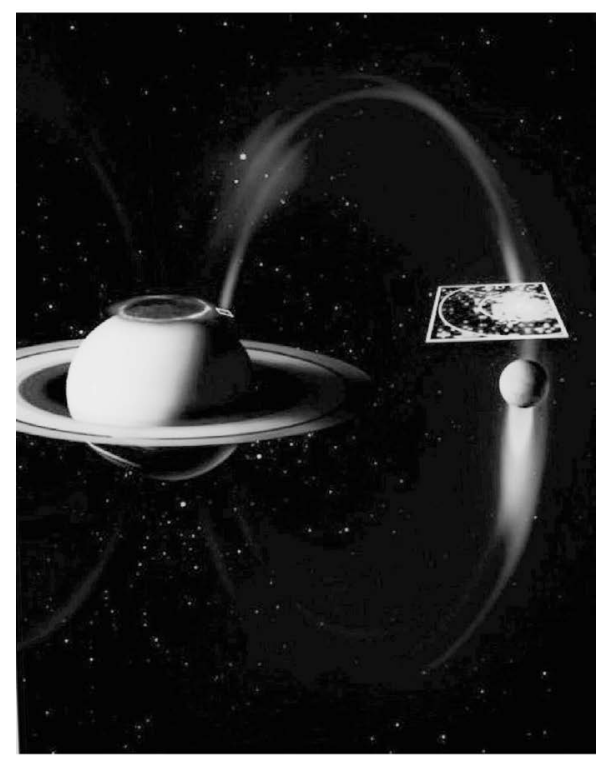

Figure 4. Electrical Circuit Between Saturn and Enceladus, https://saturn.jpl.nasa.gov/resources/5289/ Image and Title Credit: NASA/JPL/JHUAPL/University of Colorado/Central Arizona College/SSI. Electrical circuits between Saturn and Enceladus. The magnetic field also provides a path for electrons to flow as illustrated in the graphic above. There is a magnetic coupling between Saturn and Enceladus. 


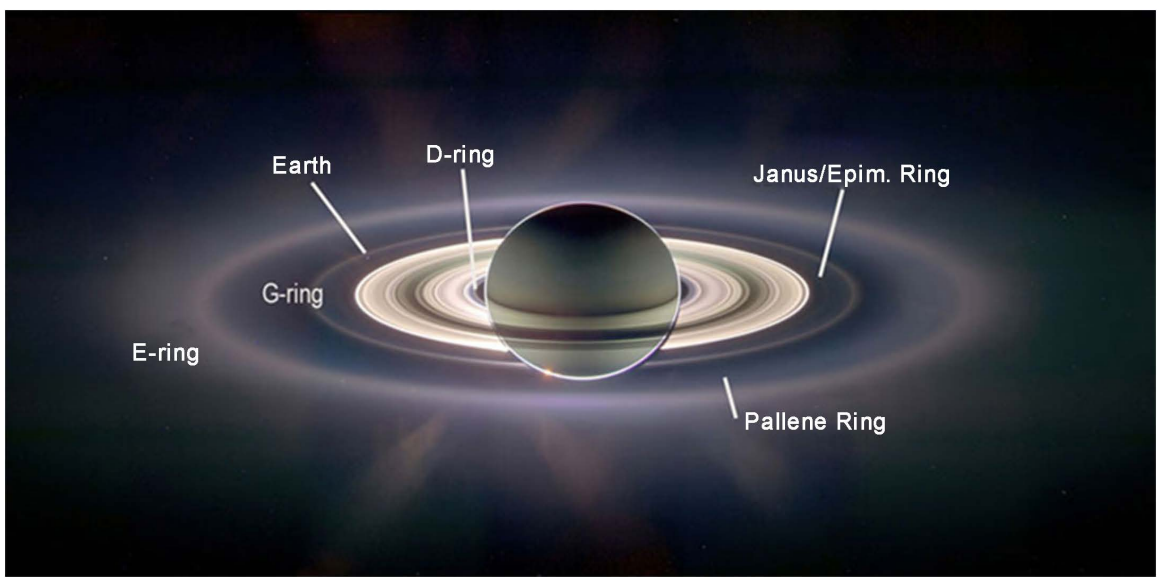

Figure 5. https://saturn.jpl.nasa.gov/resources/ Image and Title Credit:

NASA/JPL/JHUAPL/University of Colorado/Central Arizona College/SSI. Saturn rings system with E ring. Water vapor from the geysers of Enceladus supplies most of the iced particles making up an external Saturn's $E$ ring. This process is going in accordance with magnetic coupling between the Saturn and Enceladus as it shown on Figure 4 and Figure 5.

the geological activity of the satellite. It may happen at any moment of the long time existence of the Saturn system.

All experimental data of Cassini we discussed above it is a confirmation that most particles of the Saturn rings are relict of the early days of Saturn system magnetic field formation. Certainly, the rings we see today are not the same completely at the beginning of it appearance. Formation of the rings disc it is not a one-off event. The process of existence of the rings disk has a long history started from it dynamics of the initial origin from the superconducting iced particles of the protoplanetary cloud and then quantum locking and quantum trapping within magnetic equator plane. And it continues up today as it follows from the data of Cassini spacecraft.

\section{Conclusions}

Recently we coined the hypotheses that the ice matter of the Saturn rings particles or part of it may be superconducting. The above consideration has demonstrated once again how Saturn's rings initially could have formed from the superconducting icy particles of the protoplanetary cloud and in addition also with some other mechanisms of the observing phenomena. The most intriguing part of this process of the Saturn rings origin is the electromagnetic interaction of superconducting iced particles with the magnetic field of Saturn and the quantum locking and quantum trapping of them within magnetic equator plane.

Saturn rings particles are relict of the early days of the Saturn system magnetic field formation. And rings disc was formatted after appearance of the magnetic field of planet in accordance with presented above scenario. Due to the action of the additional magnetic force of the Meissner phenomenon all chaotic orbits of the particles eventually moved from the protoplanetary cloud to magnetic equator plane where there is a minimum of magnetic energy. Then particles within 
the rings disc become to be locked by the quantum locking in the horizontal direction and suppressed by external magnetic field along a meridian. As a result, each particle of the rings disk of Saturn is locked strong enough within three-dimensional magnetic well.

Magnetic well of the rings disc is a sufficiently disturbing picture of Saturn magnetic field in the area of equator. If some other superconducting particle is flying nearby the rings disc, then the magnetic well will eventually attract the particle within the disc and get locked it inside.

Thus it means we have blocked enough structure of the rings disk which is stabilized by the magnetic force of Meissner effect on the vertical direction due to the gradient of magnetic flux in the both directions from the magnetic equator and it is stabilized on the horizontal direction by the quantum pinning of the Abrikosov vortex structure for each particle.

When particles of the $B$ ring come to anomaly area of the Saturn magnetic field, they receive additional azimuth-radial movements by electromagnetic force due to Meissner effect and they start to move. In optical range it is registered as a spokes. During collision of the particles, their dot contact may generate registered wideband pulse radiation due to non-stationary Josephson phenomenon.

It is an interesting feature that the suggested mechanism of the rings formation due to electromagnetic interaction works even in case when only small fraction of the iced particles may have superconductivity. This conclusion is based on results of laboratory experiment when the small scale frozen superconductor located on the big enough size of substrate is trapped in external magnetic field and it is able to hold much more times its weight. Magnetic field is magnetized inside the superconductor. Some of the flux line becomes to be pin, they don't move. And superconductor blocks itself within magnetic field. It happened because superconductivity has quantum nature.

So, the time of initial formation of the rings disc system with thickness of a ten meters due to electromagnetic interaction of superconducting iced particles of the protoplanetary cloud with magnetic field of Saturn may be estimated as from a few thousand years up to few tens of thousands years. And this is the time of the origin, dynamics and formation of the initial state of the system of Saturn rings after appearance of the magnetic field of Saturn. During this time the chaotic orbits of the superconducting iced particles of the protoplanetary cloud which are moving around Saturn will collapse into the disc of rings.

At the same time due to a long time existence of the Saturn rings the other processes also need to be considered which could contribute to the dynamics and evolution of the rings disk. It is necessary to underline that some other sudden event like satellite coming to the Saturn and becomes to be destroyed to the debris, and coming superconducting meteorites or water eruption by the geyser of the satellite of Saturn, all of them could contribute to the dynamic and evolution of the existing structure of the rings disc matter. For example, some of the water vapor of the geyser of Enceladus falls back as "snow"; the rest escapes, and 
supplies most of the material making up Saturn's $E$ ring. We can imagine the same situation could happen some moment of time earlier with another satellite and $B$ ring.

In fact orbital resonances between Saturn, satellites, moons and rings particles play an important role in forming a specific structure of the rings system gaps and enhancing the influence of the satellites as well as the gravitational, mechanical, magnetohydrodynamic, dusty plasma and gas-plasma interactions.

It is a pleasing surprise, how many features of the Saturn rings our model of the superconductivity of the Saturn rings particles can explain. If applied to other systems, it has the potential to enrich our understanding of the space natural laboratory in general.

\section{References}

[1] Fridman, A.M. and Gorkavyi, N.N. (1999) Physics of Planetary Rings. Springer-Verlag, Berlin Heidelberg. https://doi.org/10.1007/978-3-662-03918-2

[2] Canup, R.M. (2010) Origin of Saturn's Rings and Inner Moons by Mass Removal from a Lost Titan-Sized Satellite. Nature, 468, 943-946.

https://doi.org/10.1038/nature09661

[3] Kempf, S., Altobelli, N., Srama, R., Cuzzi, J.N. and Estrada, P.R. (2017) The Age of Saturn's Rings Constrained by the Meteoroid Flux into the System. American Geophysical Union Meeting, New Orleans, 13 December 2017, 277432.

[4] Hedman, M.M. and Nicholson, P.D. (2016) The B-Ring's Surface Mass Density from Hidden Density Waves: Less than Meets the Eye? Icarus, Jan. 22, https://doi.org/10.1016/j.icarus.2016.01.007

[5] Grossman, L. (2018) Saturn's Rings Are Surprisingly Young and May Be from Shredded Moons. Science News, Jan. 20, 2918, Vol. 193, p. 7.

[6] Henry, J. (2006) Age and Fate of Saturn's Rings. Journal of Creation, 20, 123. http://www.creationconcepts.org/resources/RINGS.pdf

[7] Estrada, P.R., Durisen, R.H. and Cuzzi, J.N. (2017) Ballistic Transport: After the Cassini Grand Finale, Is There a Final Consensus on Ring Origin and Age? American Geophysical Union Meeting, New Orleans, 12 December 2017, 298112.

[8] Rowan, L., Sanchez-Lavega, A., Gombosi, T.I., Hansen, K.S., Porco, C.C., et al. (2005) Cassini at Saturn. Science, 307, 1222-1276. https://doi.org/10.1126/science.307.5713.1222

[9] Burns, J.A. and Cuzzi, J.N. (2006) Our Local Astrophysical Laboratory. Science, 312, 1753-1755. https://doi.org/10.1126/science.1114856

[10] Dougherty, M., Esposito, L. and Krimigis, T. (Eds.) (2009) Saturn from Cassini-Huygens. Springer, Dordrecht. https://doi.org/10.1007/978-1-4020-9217-6

[11] Cuzzi, J.N., Burns, J.A., Charnoz, S., Clark, R.N., Colwell, J.E., Dones, L., Esposito, L.W., Filacchione, G., French, R.G., Hedman, M.M., Kempf, S., Marouf, E.A., Murray, C.D., Nicholson, P.D., Porco, C.C., Schmidt, J., Showalter, M.R., Spilker, L.J., Spitale, J.N., Srama, R., Sremčević, M., Tiscareno, M.S. and Weiss, J. (2010) An evolving View of Saturn's Dynamic Rings. Science, 327, 1470-1475. https://doi.org/10.1126/science.1179118

[12] Tiscareno, M.S., Colin, J., Mitchell, C.S., Murray, C.D., Di Nino, D., Hedman, M.M., Schmidt, J., Burns, J.A., Cuzzi, J.N., Porco, C.C., Beurle, K. and Evans, M.W. (2013) Observations of Ejecta Clouds Produced by Impacts onto Saturn's Rings. Science, 
340, 460-464. https://doi.org/10.1126/science.1233524

[13] Safronov, V.S. (1969) Evolution of the Protoplanetary Cloud and Formation of the Earth and the Planets. Nauka Press, Moscow. (NASA Technical Translation F-677, 1972)

[14] Pospelov, A.Yu. and Tchernyi, V.V. (1995) Electromagnetic Properties Material Forecast of the Planetary Rings by the Methods of Functionally Physical Analysis. Proc. Intern. Scientific-Methodological Conf. on Innovative Design in Education, Techniques and Technologies, Volgograd State Technical University, Volgograd (Russian), 75-77.

[15] Pospelov, A.Yu., Tchernyi, V.V. and Girich, S.V. (1998) Planet's Rings: Super-Diamagnetic Model and New Course of Investigations. Proc. SPIE 42nd Annual Meeting, San Diego, CA, 27 July-1 August 1997; Small Spacecraft, Space Environments and Instrumentation Technologies, SPIE, 3116, 117-128.

[16] Pospelov, A.Yu., Tchernyi, V.V. and Girich, S.V. (1998) Possible Explanation of the Planet's Rings Behavior in the Radio and MM-Wave Range via Superdiamagnetic Model. SPIE International Symposium on Astronomical Telescopes and Instrumentation, Kona, 20-23 July 1998, No. 73.

[17] Pospelov, A.Yu., Tchernyi, V.V. and Girich, S.V. (1998) Superdiamagnetic Model of Planetary Rings Behavior in the Millimeter and Submullimeter Range. 4th International Conference on MM and SMM Waves and Applications, San Diego, 20-23 July 1998, 172-173.

[18] Girich, S.V., Pospelov, A.Yu. and Tchernyi, V.V. (1998) Radar Data Explanation via Superdiamagnetic Model of the Saturn's Rings. Bulletin of the American Astronomical Society, 30, 1043.

[19] Tchernyi, V.V., Pospelov, A.Yu. and Girich, S.G. (1998) Studies on the Rings of Saturn. The Academy for Future Science, Los Gatos.

[20] Pospelov, A.Yu., Tchernyi, V.V. and Girich, S.G. (1999) Anomalous Inversion of Polarization of Icy Satellites and Saturn's Rings: Superdiamagnetic Model. 44th SPIE Annual Meet, Denver, 18-23 July 1999, Vol. 3754, 329-333.

[21] Pospelov, A.Yu., Tchernyi, V.V. and Girich, S.G. (1999) Are Saturn Rings Superconducting? University of Alabama, NASA Marshall Space Flight Center, Huntsville.

[22] Pospelov, A.Yu., Tchernyi, V.V. and Girich, S.G. (2000) Are Saturn's Rings Superconducting? Progress in Electromagnetic Research Symposium, MIT, Cambridge, 1158 .

[23] Tchernyi, V.V. (2002) Possible Superconductivity of the Saturn Rings. University of Hawaii, Institute for Astronomy, Honolulu.

[24] Tchernyi, V.V. and Pospelov, A.Yu. (2005) Possible Electromagnetic Nature of the Saturn's Rings: Superconductivity and Magnetic Levitation. Progress in Electromagnetic Research, 52, 277-299. https://doi.org/10.2528/PIER04082801

[25] Tchernyi, V.V. and Pospelov, A.Yu. (2005) About Possible Electromagnetic Nature of the Planetary Rings: Magnetic Levitation of Superconducting Rings of Saturn. Fizika Volnovykh Processov I Radiotehnicheskih System, 8, 4-16. (In Russian)

[26] Tchernyi, V.V. and Chensky, E.V. (2005) Electromagnetic Background for Possible Magnetic Levitation of the Superconducting Rings of Saturn. Journal of Electromagnetic Waves and Applications, 19, 1997-2006.

https://doi.org/10.1163/156939305775570440 
[27] Tchernyi, V.V. and Chensky, E.V. (2006) Movements of the Protoplanetary Superconducting Particles in the Magnetic Field of Saturn Lead to the Origin of Rings. Geoscience and Remote Sensing Letters, 2, 445-446.

[28] Tchernyi, V.V. (2006) About Possible Role of Electromagnetism and Superconductivity for the Origin of Saturn Rings. Prikladnaya Fizika (Applied Physics), 5, 10-16. (In Russian)

[29] Tchernyi, V.V. and Pospelov, A.Yu. (2007) About Hypothesis of the Superconducting Origin of the Saturn's Rings. Astrophysics and Space Science, 307, 347-356. https://doi.org/10.1007/s10509-006-9054-7

[30] Tchernyi, V.V. (2009) Origin of the Saturn Rings: Electromagnetic Model of the Sombrero Rings Formation. In: Denis, J.H. and Aldridge, P.D., Eds., Space Exploration Research, Nova Science Publishers, Hauppauge, Chapter 11, 261-275.

[31] Tchernyi, V.V. (2009) Origin of the Saturn Rings: Electromagnetic Model of the Sombrero Rings Formation. Journal of Magnetohydrodynamics, Plasma and Space Research, 14, 385-398.

[32] Tchernyi, V.V. (2013) Could Superconductivity Contribute to the Saturn Rings Origin? Journal of Modern Physics, 4, 17-23. https://doi.org/10.4236/jmp.2013.46A005

[33] Cherny, V.V. (2013) The Saturn Rings Origin: Contribution of Electromagnetism (to the Unified Theory of the Origin of Planetary Rings). American Journal Astronomy and Astrophysics, 1, 15-22. https://doi.org/10.11648/j.ajaa.20130102.11

[34] Tchernyi, V.V. (2013) About Role of Electromagnetism to the Saturn Rings Origin-To the Unified Theory of the Planetary Rings Origin. International Journal of Astronomy and Astrophysics, 3, 412-420. https://doi.org/10.4236/ijaa.2013.34049

[35] Tchernyi, V.V. and Pospelov, A.Yu. (2015) Modern Physics Solution of the Saturn Rings Origin Problem-Electromagnetism and Superconductivity (The Possibility of the Unified Theory of the Planetary Rings Origin). International Journal of Modern Physics and Application, 5, 65-72.

http://www.aascit.org/journal/archive?journalId=909\&issueId=9090205

[36] Tchernyi, V.V. and Pospelov, A.Yu. (2018) Superconductivity of Saturn Rings: Quantum Locking, Rings Disc Thickness and Its Time Creation. Journal of Modern Physics, 9, 419-432. https://doi.org/10.4236/jmp.2018.93029

[37] Alfven, H. (1981) Solar System History as Recorded in the Saturnian Ring Structure. Astrophysics and Space Science, 97, 79-94. https://doi.org/10.1007/BF00684612

[38] Bednorz, J.G. and Müller, K.A. (1986) Possible High Tc Superconductivity in the Ba-La-Cu-O System. Zeitschrift für Physik B, 64, 189-193. https://doi.org/10.1007/BF01303701

[39] Babushkina, G.V., Kobelev, L.Ya., Yakovlev, E.N. and Babushkin, A.N. (1986) Superconductivity of Ice under High Pressure. Physics of Solid State, 28, 3732-3734. (In Russian)

[40] Yen, F. and Gao, T. (2015) Dielectric Anomaly in Ice near 20K; Evidence of Macroscopic Quantum Phenomena. The Journal of Physical Chemistry Letters, 6, 2822-2825. https://doi.org/10.1021/acs.jpclett.5b00797

[41] Côté, M., Grossman, J.C., Cohen, M.L. and Louie, S.G. (1998) Electron-Phonon Interactions in Solid C36. Physical Review Letters, 81, 697-700. https://doi.org/10.1103/PhysRevLett.81.697

[42] Wampler, J., et al. (2018) Natural Superconductivity Observed in Meteorites above 5 K. American Physical Society March Meeting, Los Angeles, 5-9 March 2018. 
[43] Deutscher, G., Azoulay, M., Almog, B. and Deutscher, B. (2011) Quantum Levitation, Quantum Locking, Quantum Trapping. ASTC Conference, Maryland, 15-18 October 2011.

http://library.fora.tv/2012/10/25/Dr_Boaz_Almog_Quantum_Levitation

[44] Abrikosov, A.A. (1957) On the Magnetic Properties of Superconductors of the Second Group. Soviet Physics JETF, 5, 1174-1182.

[45] Essmann, U. and Träuble, H. (1967) The Direct Observation of Individual Flux Lines in Type II Superconductors. Physics Letters A, 24, 526-527. https://doi.org/10.1016/0375-9601(67)90819-5

[46] Tonomura, A., et al. (2001) Observation of Individual Vortices Trapped along Columnar Defects in High-Temperature Superconductors. Nature, 412, 620-622. https://doi.org/10.1038/35088021

[47] Abrikosov, A.A. (2003) Type II Superconductors and the Vortex Lattice. Nobel Lecture.

https://www.nobelprize.org/nobel_prizes/physics/laureates/2003/abrikosov-lecture. pdf

[48] Sigal, I.M. and Tzaneteas, T. (2013) On Stability of Abrikosov Lattices. https://arxiv.org/abs/1308.5446v1

[49] Sigal, I.M. and Tzaneteas, T. (2016) On Stability of Abrikosov Vortex Lattices. https://arxiv.org/abs/1308.5446

[50] Hansen, C.J., Esposito, L., Stewart, A.I.F., Colwell, J., Hendrix, A., Pryor, W., Shemansky, D. and West, R. (2006) Enceladus' Water Vapor Plume. Science, 311, 1422-1425. https://doi.org/10.1126/science.1121254

[51] Spencer, J.R. and Nimmo, F. (2013) Enceladus: An Active Ice World in the Saturn System. Annual Review of Earth and Planetary Sciences, 41, 693-717.

[52] Dyches, P., Brown, D., et al. (2014) Cassini Spacecraft Reveals 101 Geysers and More on Icy Saturn Moon. NASA.

[53] NASA (2015) Jump up to: Icy Tendrils Reaching into Saturn Ring Traced to Their Source.

[54] NASA (2006) Jump up to: Ghostly Fingers of Enceladus. NASA/JPL/Space Science Institute.

[55] Meyer, J. and Wisdom, J. (2007) Tidal Heating in Enceladus. Icarus, 188, 535-539. https://doi.org/10.1016/j.icarus.2007.03.001 